\title{
NEW FEATURE PRESERVING NOISE REMOVAL ALGORITHM BASED ON THE DISCRETE COSINE TRANSFORM AND THE A PRIOR KNOWLEDGE OF PIXEL TYPE
}

\author{
H. C. Yung ${ }^{*} \&$ H. S. Lai ${ }^{\star *}$ \\ Department of Electrical \& Electronic Engineering \\ The University of Hong Kong \\ Pokfulam Road, HONG KONG \\ *Email : nyung@hkueee.hku.hk \\ **Email : hslai@hkueee.hku.hk
}

\begin{abstract}
In this paper, a new Corrupted-Pixel-Identification (CPI) based estimation filter is presented. The method is especially useful for filtering clustered noise. After the corrupted pixels are identified by the CPI algorithm, the noisy subimage centered on a corrupted pixel is transformed into its Discrete Cosine Transform (DCT) domain where the transformed subimage is approximated by its DC coefficient only. With the knowledge of the number of corrupted pixels in the subimage from the CPI algorithm, an estimation of the noise distribution can be made, from which the DC coefficient of the restored subimage can be determined. Hence, noise filtering is achieved. From the experimental results, we can show that the CPI-based estimation filter has three desirable characteristics: 1) it has superior noise removal performance over the conventional median filter and CPI-based median filter in filtering clustered noise; 2 ) it has good feature preserving property (better than conventional filters); and 3) the computing speed of the filter is almost three times faster than the conventional median filter.
\end{abstract}

\section{INTRODUCTION}

Noise filtering is a technique commonly employed in digital image processing applications where the images are degraded by randomly populated impulses in the spatial domain. Additive white impulses are often the consequence of poor input sampling and/or interference from an external source. If the noise distribution is known exactly, the original image can be recovered from the noisy image completely in the additive case. However, this is often not possible and therefore, estimation of the noise distribution and intensity is commonly carried out in spatial filtering.

Over the years, many noise filtering algorithms in the spatial domain have been developed. Typical examples of these filters are the median filter[1,2,4], averaging filter[3,4], sigma filter[4], box filter[1,4] and general rank filter[1]. These conventional spatial filtering algorithms have a common problem: they introduce certain degree of line and edge distortion to the recovered image[5], which manifests itself as slight blurring or smoothing as a result. One of the reasons for this distortion is the filtering without discrimination. In other words, every pixel in the image is subjected to the same neighbourhood operation uniquely defined by the operating algorithm. The implementation of the this kind of filter is usually simple and straight forward, although it could be time wasting. This is because not all the pixels in the image are corrupted, processing all the pixels means waste of time in filtering uncorrupted pixels. In addition, filtering uncorrupted pixels would vary image features that perhaps is unacceptable in applications such as video communications.

In contrast to filtering without discrimination, another class of filtering algorithms based on the idea of "filtering with discrimination" have been developed[6,7]. In this class of algorithms, the basic concept is that pixels are being classified into one of the two classes: corrupted or uncorrupted; before the corrupted pixels are being filtered by a conventional spatial filter or a specially designed algorithm making use of the a priori knowledge of the pixel types. Figure 1 illustrates the conceptual diagram of the CPI algorithm. This algorithm offers a substantial saving in computing time[8]. Furthermore, by not filtering the uncorrupted pixels, the CPI-based filters have features preserving property that is better than the other conventional algorithms.

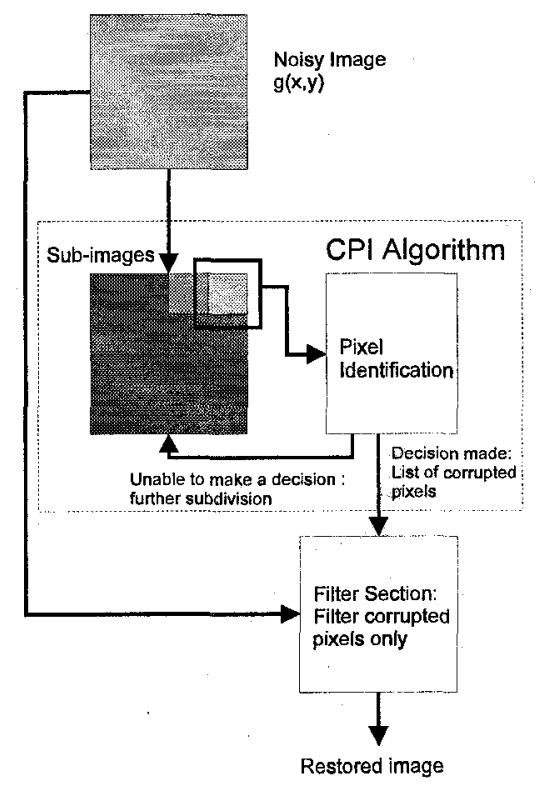

Figure 1 : Conceptual diagram of the $\mathrm{CPI}$ algorithm

In the case of the CPI-median filter, the list of corrupted pixel generated by the CPI algorithm is passed to the Filter Section where a median filter is used to perform the standard filtering operation. In this case, no attempt has been made on 
how the noise removing quality and features preserving quality may be improved by using the a priori knowledge of the pixel type during the filtering process. It is the intention of this paper to explore this particular aspect in conjunction with the CPI algorithm, and investigate the effect of filtering a corrupted pixel by considering only the corrupted pixels in its reighbourhood.

In this paper, a novel filtering algorithm using the a priori knowledge of the pixel type to estimate the noise distribution and intensity in a subimage is presented. The subimage centered on the corrupted pixel is transform into its Discrete Cosine Transform (DCT) domain where the transformed subimage is approximated by its DC coefficient only and an estimation of the noise distribution is made by combining the knowledge of the number of corrupted pixels in the subimage and the pixel intensity of the noise term. This enables the DC coefficient of the restored image in the DCT domain to be determined and hence noise intensity in the spatial domain can be determined. Simple subtraction operation is then carried out to remove the noise component from the corrupted pixel.

In the next section, the concept and philosophy are presented as well as the estimation algorithm. The experimental results of the CPI-estimation algorithm are presented in section 3. In that section, the noise removal capability, feature preserving property and computing speed of the CPI-estimation filter are evaluated. The last section is the conclusion, which gives an overall comment on the CPI-estimation filter.

\section{CPI-based Estimation Filter Algorithm}

\subsection{Concept and Philosophy}

The philosophy of this algorithm is that if we have the a prior knowledge of the pixel types, then the noise distribution can be estimated from the noisy image. In the case of the image corrupted by additive random noise, the reconstructed image can be obtained simply by subtracting the noise term from the noisy image. A high noise removal capability and high original feature preserving rate are aimed in the proposed filter algorithm

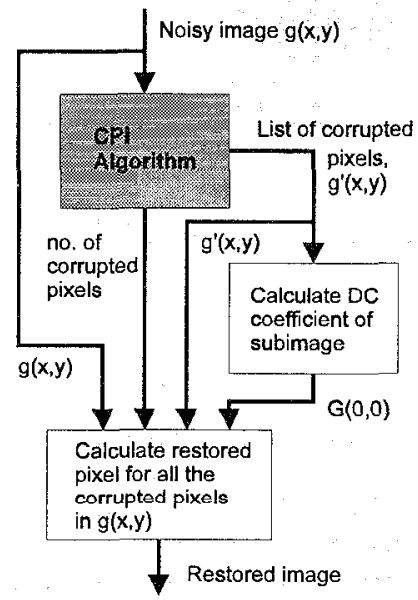

Figure 2 : Conceptual diagram of CPI-estimation Filter
Figure 2 depicts the conceptual diagram of the estimation filtering algorithm where a list of corrupted pixels $g^{\prime}(x, y)$ classified by the CPI algorithm is passed to the filter. Then the DC coefficient $G(0,0)$ in the DCT domain of the subimage centered at the corrupted pixel is calculated. The number of corrupted pixels, noisy image $g(x, y)$, list of corrupted pixels $g^{\prime}(x, y)$ and the DC coefficient $G(0,0)$ are used to estimate the noise distributions in each subimage and the estimated noise distributions are used to determine the DC coefficient of the restored image $\hat{F}(0,0)$. Finally, the noise term is calculated from $G(0,0)$ and $\hat{F}(0,0)$. The restored image $\hat{f}(x, y)$ is simply obtained by subtracting the noise term from $g(x, y)$.

\subsection{Algorithm}

Let us define the following terms :

$\begin{array}{ll}f(x, y) & : \text { Original image } \\ \eta(x, y) & : \text { Additive noise term } \\ g(x, y) & : \text { Noisy image } \\ \hat{f}(x, y) & : \text { Restored image } \\ \varepsilon & : \text { Approximated noise intensity }\end{array}$

$F_{2 N+1}(0,0):$ DC term of $F(u, v)$ over a $(2 \mathrm{~N}+1)^{2}$ subimage

$G_{2 N+1}(0,0):$ DC term of $G(u, v)$ over a $(2 \mathrm{~N}+1)^{2}$ subimage

$\hat{F}_{2 N+1}(0,0):$ DC term of $\hat{F}(u, v)$ over a $(2 \mathrm{~N}+1)^{2}$ subimage

$S \quad:$ Number of corrupted pixels (from CPI) inside a $(2 \mathrm{~N}+1)^{2}$ subimage

The DCT of $f(x, y)$ and its inverse are :

$$
\begin{aligned}
F(u, v) & =\frac{2}{2 N+1} C(u) C(v) \times \\
& {\left[\sum_{x=-N}^{N} \sum_{y=-N}^{N} f(x, y) \cos \frac{(2 x+1) u \pi}{2(2 N+1)} \cos \frac{(2 y+1) v \pi}{2(2 N+1)}\right] } \\
f(x, y) & =\frac{2}{2 N+1}\left[\sum_{u=-N}^{N} \sum_{v=-N}^{N} C(u) C(v) \times\right. \\
& \left.F(u, v) \cos \frac{(2 x+1) u \pi}{2(2 N+1)} \cos \frac{(2 y+1) v \pi}{2(2 N+1)}\right]
\end{aligned}
$$

The DC coefficient of $F(u, v)$ when $u=0$ and $v=0$ over a $(2 \mathrm{~N}+1)^{2}$ subimage and its inverse are given by:

$$
\begin{aligned}
& F_{2 N+1}(0,0)=\frac{1}{(2 N+1)}\left[\sum_{x=-N}^{N} \sum_{y=-N}^{N} f(x, y)\right] \\
& f(x, y)=\frac{F_{2 N+1}(0,0)}{(2 N+1)}
\end{aligned}
$$

The noisy image $g(x, y)$ is the sum of the original image $f(x, y)$ and the additive noise $\eta(x, y)$, i.e.

$$
g(x, y)=f(x, y)+\eta(x, y)
$$

where the DC coefficient of $g(x, y)$ equals to :

$$
G_{2 N+1}(0,0)=\frac{1}{2 N+1}\left\{\sum_{x=-N}^{N} \sum_{y=-N}^{N} f(x, y)+\sum_{x=-N}^{N} \sum_{y=-N}^{N} \eta(x, y)\right\}
$$

Assume all the additive noise pixels within the filtering window have approximately the same intensity $\varepsilon$, then the intensity of 
the noise pixels can be estimated by

$$
\eta(x, y)= \begin{cases}\varepsilon & \text { if } g(x, y) \text { is corrupted } \\ 0 & \text { otherwise }\end{cases}
$$

This assumption is designed to deal with white impulse noise. For other types of noise distributions, equation (5) may be generalised to the average value or the sum of $\eta(x, y)$ over the $(2 \mathrm{~N}+1)^{2}$ subimage.

If there are $S$ corrupted pixels within the subimage, then equation (4) becomes:

$$
G_{2 N+1}(0,0)=F_{2 N+1}(0,0)+\frac{S \varepsilon}{2 N+1}
$$

From equation (6), if the corrupted pixel at the center of the subimage is restored, the number of corrupted pixels would become $S-1$. Therefore, the DC coefficient of the restored image becomes:

$$
\begin{aligned}
\hat{F}_{2 N+1}(0,0) & =F_{2 N+1}(0,0)+\frac{(S-1) \varepsilon}{2 N+1} \\
& =G_{2 N+1}(0,0)-\frac{\varepsilon}{2 N+1}
\end{aligned}
$$

Let the approximation of noise intensity be:

$$
\varepsilon=g(x, y)-f(x, y)
$$

Substituting equations $(2 b) \&(8)$ into (6) and rearranging, we have

$$
F_{2 N+1}(0,0)=\frac{G_{2 N+1}(0,0)-\frac{S}{2 N+1} g(x, y)}{\left\{1-\frac{S}{(2 N+1)^{2}}\right\}}
$$

By substituting equations (2b), (8) \& (9) into (7) and rearranging, the DC coefficient of $\hat{F}_{2 N+1}(0,0)$ becomes:

$$
\begin{aligned}
\hat{F}_{2 N+1}(0,0)= & G_{2 N+1}(0,0) \cdot\left[1+\frac{1}{(2 N+1)^{2}-S}\right]- \\
& g(x, y) \cdot\left[\frac{2 N+1}{(2 N+1)^{2}-S}\right]
\end{aligned}
$$

Since $g(x, y)$ is given and $S$ can be obtained from the CPI algorithm, $\hat{F}_{2 N+1}(0,0)$ can be determined from equation $(10)$. As a result, the restored image $\hat{f}(x, y)$ can be deduced from equation (2b).

\section{EXPERIMENTAL RESULTS}

\subsection{Performance Evaluation}

In this paper, the image "casino ticket" with 256 grey levels and having a spatial dimension of $227 \times 533$ is used as the test sample. This image has coast features, such as the title, icons and the large numerals on the right hand side; and also fine features, such as the horizontal lines and the small characters. Noisy images varying from $\mathrm{SNR}=50 \mathrm{~dB}$ to $-50 \mathrm{~dB}$ are tested by the conventional median filter, the CPI-median filter and the proposed CPI-estimation filter. Subjective measurement is based on visual inspection and objective measurement is based on the mean-square error (MSE) which is given by equation (11)

$$
\sigma_{m s}=\frac{\sum_{y=1}^{H} \sum_{x=1}^{W}[f(x, y)-\hat{f}(x, y)]^{2}}{W \times H}
$$

where $W \times H$ is the dimension of the image.

Furthermore, the computing speed of the above algorithms are compared.

\subsection{Noise Removal Capability}

The restored images from the noisy image with SNR equals to $-50 \mathrm{~dB}$ by different filters are shown in Figure 3. From the images, it is quite clear that the CPI-estimation filter is more effective in removing clustered noise where clustered noise often appear in low SNR images while the other two filters fail to remove most of the clustered noise.

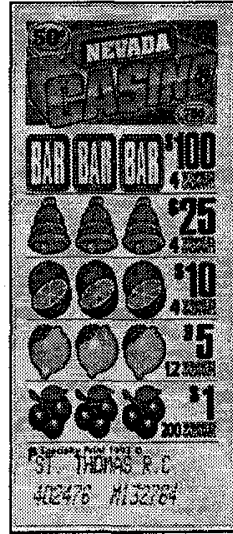

(a)

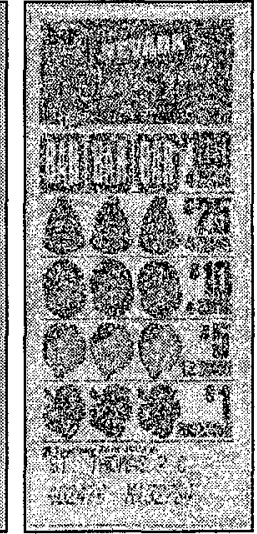

(b)

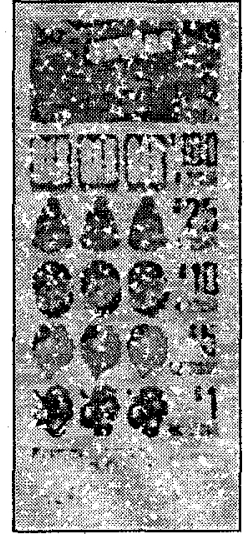

(c)

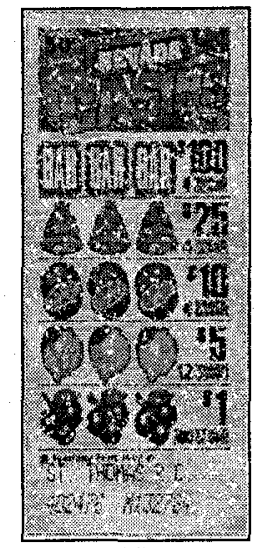

(d)

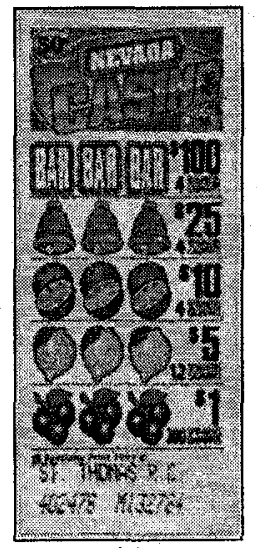

(e)
Figure 3 : (a) Original image; (b) Noisy image ( $S N R=-50 \mathrm{~dB}$ );

(c) Restored by median filter; (d) Restored by CPI-median filter; (e) Restored by CPI-estimation filter.

Figure 4 plots the MSE for all the three cases. From the figure, the CPI-based filters are more effective than the median filter over the whole range. While the CPI-estimation filter has better performance than the CPI-median filter for $\mathrm{SNR}<10 \mathrm{~dB}$. In addition, the MSE of the CPI-estimation filter increases fairly slightly as SNR decreases. 


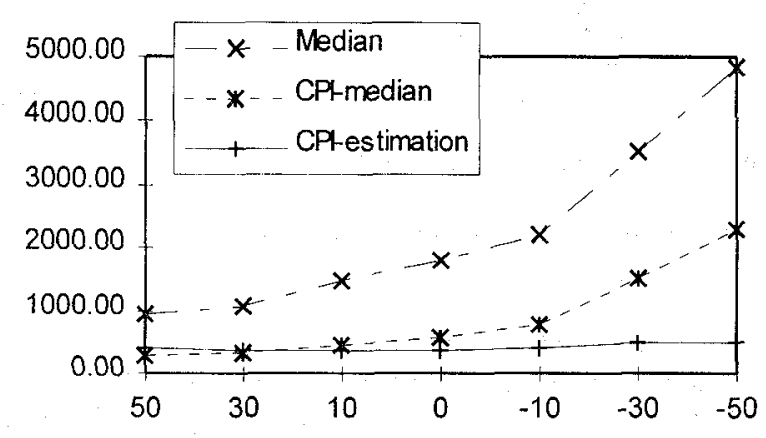

Figure 4 : MSE of the three filters versus SNR

\subsection{Feature Preserving Property}

To investigate the feature preserving property, the different filters are applied to the original uncorrupted image and the MSE of the filtered images are tabulated in table 1.

\begin{tabular}{|c|c|c|}
\hline Median & CPI-median & CPI-estimation \\
\hline 920.1 & 285.57 & 421.21 \\
\hline
\end{tabular}

Table 1 : Comparison of MSE between different filters

From the table, CPI-median gives the lowest MSE, this means that the CPI-median filter has better feature preserving property followed by the CPI-estimation filter and the median filter is the poorest. The filtered images are shown in Figure 5, in which both the CPI-based filters retain most of the detail information while the median filter blurred those horizontal lines and small characters in the image.

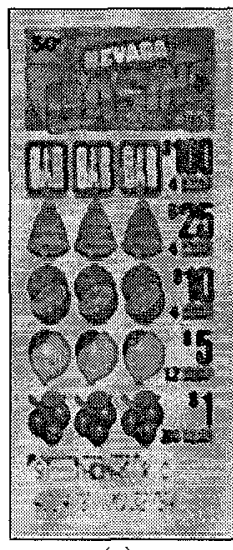

(a)

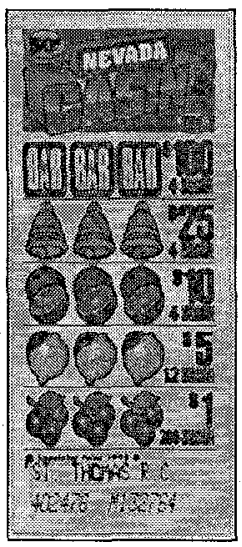

(b)

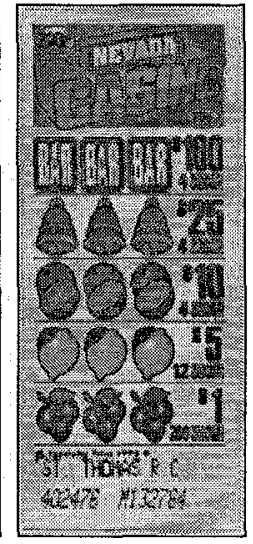

(c)
Figure 5 : (a) Filtered by median filter; (b) Filtered by $\mathrm{CPI}$-median filter; (c) Filtered by CPI-estimation filter

\subsection{Computing Speed}

The CPI-estimation filter employs simple arithmetic operations which are faster than sorting operations used in the median filter. Table 2 depicts the computing time of the three filters running on a SGI workstation(Indy $133 \mathrm{MHz}, \mathrm{R} 4600 \mathrm{PC}$ ).

Obviously, the median filter takes constant computing time for all cases of SNR because of its filtering without discrimination philosophy. The CPI-median filter is around 1.6 times faster than median filter. For the CPI-estimation filter, which is the fastest amongst the three filters, the average computing time is 1.46 seconds which is almost three times faster than the median filter. It should also be noted that the computing time of the two CPI-based filters varies according to the number of corrupted pixels identified

\begin{tabular}{|c|c|c|c|}
\hline SNR(dB) & Median & CPI-median & CPI-estimation \\
\hline 50 & $4.7 \mathrm{~s}$ & $2.5 \mathrm{~s}$ & $1.6 \mathrm{~s}$ \\
\hline 30 & $4.7 \mathrm{~s}$ & $2.1 \mathrm{~s}$ & $1.4 \mathrm{~s}$ \\
\hline 10 & $4.7 \mathrm{~s}$ & $2.5 \mathrm{~s}$ & $1.6 \mathrm{~s}$ \\
\hline 0 & $4.7 \mathrm{~s}$ & $2.2 \mathrm{~s}$ & $1.4 \mathrm{~s}$ \\
\hline-10 & $4.7 \mathrm{~s}$ & $2.1 \mathrm{~s}$ & $1.3 \mathrm{~s}$ \\
\hline-30 & $4.7 \mathrm{~s}$ & $2.2 \mathrm{~s}$ & $1.4 \mathrm{~s}$ \\
\hline-50 & $4.7 \mathrm{~s}$ & $2.4 \mathrm{~s}$ & $1.5 \mathrm{~s}$ \\
\hline
\end{tabular}

Table 2 : Computing times required for different filters

\section{CONCLUSIONS}

CPI-based filters generally outperform the conventional median filter algorithm in terms of objective MSE, subjective visual inspection and computing speed. CPI-estimation filter is particularly useful for filtering heavily corrupted images as well as having good features preserving property. In addition, the computing speed of 1.46 times faster than CPI-median filter and 3 times faster than the conventional median filter, making it the fastest in this class. In conclusion, filtering with a priori knowledge of the corrupted pixels is more effective than the conventional filter methods.

\section{REFERENCES}

[1] John C. Russ, "The Image Processing Handbook", 2/e, IEEE Press, 1995.

[2] Milan Sonka, Vaclav Hlavac \& Roger Boyle, "Image Processing, Analysis and Machine Vision", Chapman \& Hall Computing, 1994.

[3] Robert, J. Schalkoff, "Digital Image Processing and Computer Vision", John Wiley \& Sons, 1989.

[4] Zheung Yi, "Image analysis, modeling, enhancement, restoration, feature extraction and their application in nondestructive evalution and radio astronomy", Ph.D. Thesis, Dept of EE, Iowa State University, USA, 1987.

[5] J. E. Hall \& J. D. Aetrey, "Real-time image enhancement using $3 \times 3$ pixel neighbourhood operation functions", Selected Papers on Digital Image Processing, vol. MS17, edited by Mohan M. Trivedi, SPIE Optical Engineering Press, pp. 595-598, 1990.

[6] N. H. C. Yung \& A. H. S. Lai, "A Novel Filter Algorithm for Removing Impulse Noise in Digital Images", SPIE Proc. on VCIP'95, vol. 2501, pp. 210-220, 1995.

[7] N. H. C. Yung, A. H. S. Lai \& K. M. Poon, "Modified CPI Filter Algorithm for Removing Salt-and-Pepper Noise in Digital Images", SPIE Proc. on VCIP'96, vol. 2727, pp. 1439-1449, 1996

[8] C. F. Yung, "Performance Evaluation of Class of CPI Algorithms", University of Hong Kong, Dept. EEE Training Report, October, 1995. 